\title{
Thermal conductivity measurements at low temperatures ${ }^{\dagger}$
}

\author{
P BALAYA, H S JAYANNA*, HEMANT JOSHI, G SUMANA, \\ V G NARASIMHA MURTHY, V PRASAD and S V SUBRAMANYAM \\ Department of Physics, Indian Institute of Science, Bangalore 560012. India \\ *At present: Department of Physics, Kuvempu University, B.R. Project, Shimoga 577115 , \\ India
}

\begin{abstract}
We describe briefly the experimental facilities developed for the measurement of thermal conductivity of solids in the temperat ure range $10 \mathrm{~K}-300 \mathrm{~K}$. Different techniques have been used for the determination of thermal conductivity, depending on the relaxation time of the system under investigation. Measurements on stainless steel 304, using steady state and non-steady state methods are presented. Values of thermal conductivity obtained by both these methods agree to each other and are consistent with those reported earlier.
\end{abstract}

Keywords. Low temperature; thermal conductivity; heat capacity; pulse method.

\section{Introduction}

Accurate thermal conductivity measurements are important in the development and verification of theories of electronic and lattice wave propagation in metals and non-metals. This paper presents a brief description of the design and fabrication of a cryostat, developed for the measurement of thermal conductivity of solids in the temperature range $10 \mathrm{~K}-300 \mathrm{~K}$. These facilities have been established in connection with the work undertaken to investigate the thermal conductivity $(\lambda)$ behaviour of solid and cellular polymers at low temperatures and the effect of $\gamma$-irradiation on $\lambda$ of solid polymers.

\section{Experimental}

For the investigation of thermal conductivity at low temperatures, we have developed two facilities. The first facility is an indigenous bath type cryostat for the measurement in the temperature range $4 \cdot 2 \mathrm{~K}-300 \mathrm{~K}$. The general form of the cryostat is shown in figure 1 , where the whole cryostat along with the sample chamber is immersed inside a liquid helium dewar (Oxford Instruments). The second facility is an experimental set-up fabricated, for using it along with a closed cycle refrigerator (Janis, Model: CCS-150). This facility enables the measurement of thermal conductivity under steady state condition over the temperature range $10 \mathrm{~K}-325 \mathrm{~K}$, with helium gas being used as a refrigerant.

The sample holder used in both - cryostat and closed cycle refrigerator (CCR)contain two heating elements: one serves to set the temperature of the sample, primary heater, and other to establish a thermal gradient along the length of the sample, secondary heater. The sample is thus clamped in both cases in a usual manner, with one end of the specimen in good thermal contact with the cold head of the cryostat/CCR

\footnotetext{
${ }^{\dagger}$ Paper presented at the poster session of MRSI AGM VI, Kharagpur, 1995
} 


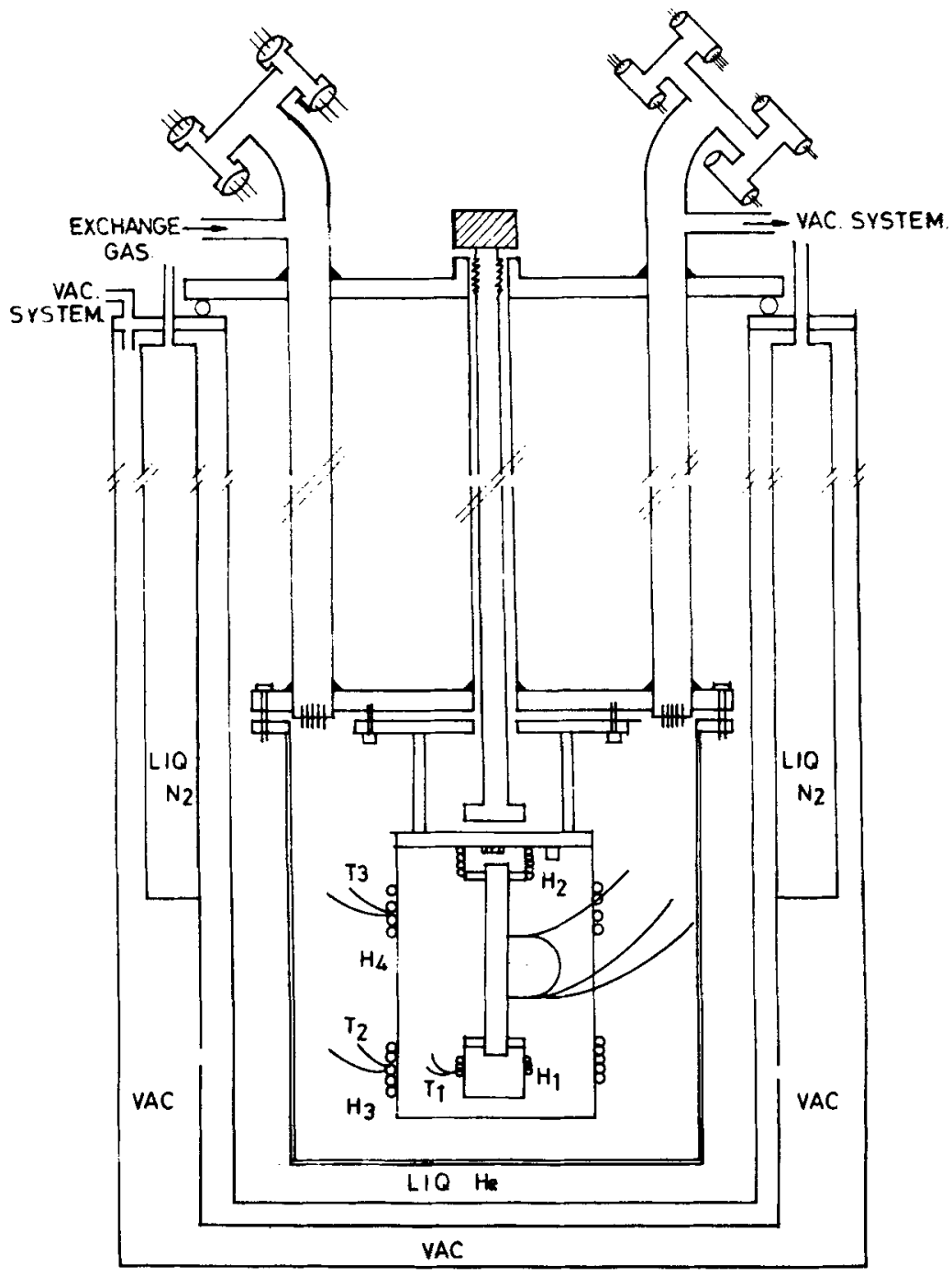

Figure 1. Schematic diagram of the cryostat.

and the other end with an electrical heater (secondary heater). Both primary and secondary heater blocks, made of electrolytic copper, are wrapped with No. 36 AWG nichrome heater wire with 30 and $120 \Omega$ respectively. The power input to these heaters are controlled using an electronic circuit (not shown) in the range $10^{-4} \mathrm{~W}-1 \mathrm{~W}$ depending on the average temperature of sample.

Differential and absolute temperatures of the sample are measured with No. 49 AWG copper-constantan thermocouples with ice as reference point. Five differential thermocouples are used which can be mounted along the length of the sample to measure the thermal gradient as a function of distance. Heat losses by gas convection and conduction are made negligible by evacuating the region surrounding the sample to $10^{-5}$ torr. Losses by radiation are minimized by enclosing the sample with nickel 
plated copper cover shorted thermally with the cold head. The thermal gradient between sample and shield can be adjusted to be within $1 \mathrm{~K}$ with the help of the nichrome wire wound on the thermal shield.

The block diagram (figure 2) shows the arrangement of whole experimental set-up interfaced through a computer, so that the thermo e.m.f. generated in thermocouples corresponding to absolute and differential temperatures, measured using DMM (Keithley, Model: 193A) can be collected and stored for further data analysis.

\section{Measurement techniques and methods of analysis}

The thermal conductivity of metals and insulators have been measured in both indigenous cryostat and closed cycle refrigerators using the following techniques: (i) steady state, (ii) modified steady state and (iii) non-steady state.

In the steady state method, the sample is subjected to a temperature profile and allowed to reach an equilibrium state. The thermal conductivity $(\lambda)$ is determined for a known quantity of heat flow $(\dot{Q})$ per unit area, by measuring the temperature gradient $(\Delta T)$ across the length $(\Delta x)$ of the sample. Thus $\lambda$ can be calculated using one dimensional Fourier's heat flow equation

$$
\lambda=-(\dot{Q} / A)(\Delta x / \Delta T) \text {. }
$$

In the modified steady state method, the temperature is measured point by point along the length of the sample using five differential thermocouples, after establishing

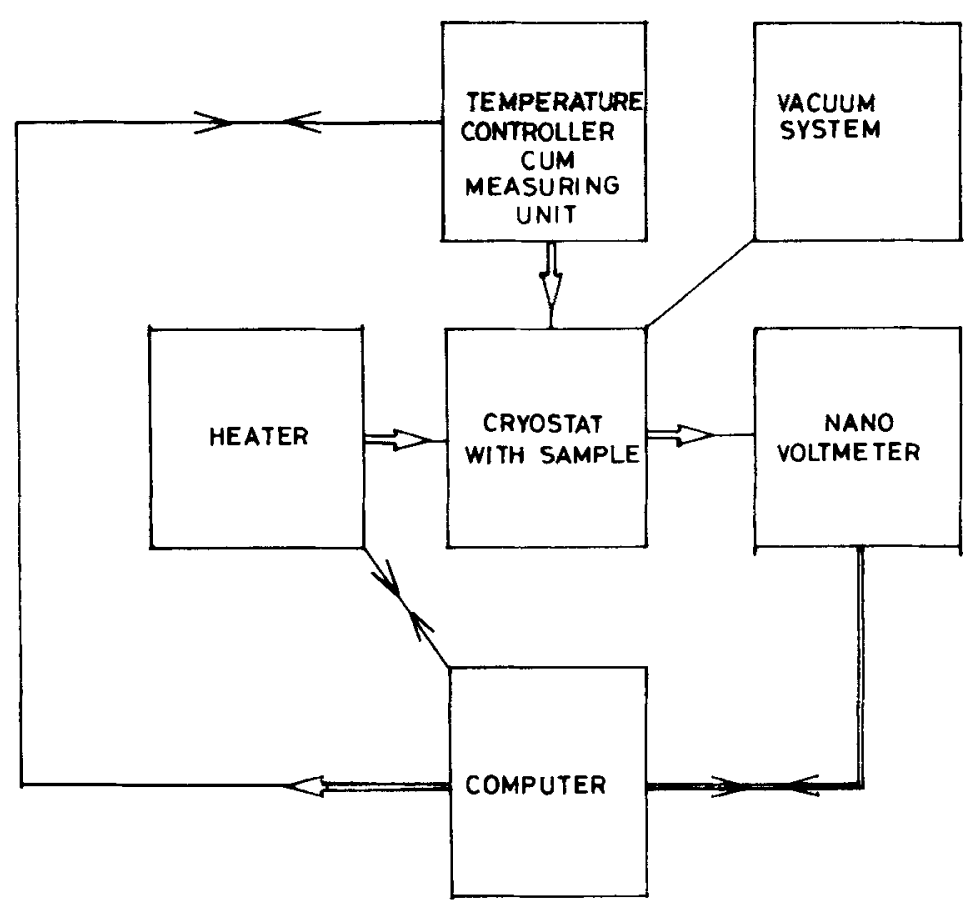

Figure 2. Block diagram of the experimental set up. 
a steady thermal gradient. Thermal conductivity as a function of temperature can be determined from this measured temperature profile $T(x)$.

In the non-steady state (pulse) method (Maldonado 1992), the temperature of the sample is slowly drifted while the heat current which generates the thermal gradient is pulsed with a square wave-generated from a Keithley programmable current source (Keithley, Model: 220). Schematic presentation of this pulse method is shown in figure 3, where the time dependence of temperature gradient $(\Delta T)$ takes an exponential shape.

The thermal conductance $(k)$ is calculated from the peak to peak amplitude of the thermal gradient $\left(\Delta T_{\mathrm{pp}}\right)$ and by solving simultaneously, the following expressions using numerical iteration

$$
\begin{aligned}
& k=\left(\dot{Q} / \Delta T_{\mathrm{pp}}\right) \tan h(k \tau / 2 C), \\
& C=\left[2 \dot{Q} /(\dot{\delta} \Delta T / \delta t)_{\mathrm{pp}}\right][1+\exp (-k \tau / C)]^{-1},
\end{aligned}
$$

where $k$ is the thermal conductance of the sample $(k=\lambda \cdot A / \Delta x), C$ the heat capacity of the heater source, $\tau$ the half width of the pulsed current, $\Delta T_{\mathrm{pp}}$ the peak to peak amplitude of temperature gradient and $(\delta \Delta T / \delta t)_{\mathrm{pp}}$ the peak to peak amplitude of $(\delta \Delta T / \delta t)$.

It is to be noted that the thermal conductance $(k)$ should be multiplied by the cell constant $(\Delta x / A)$ to obtain the thermal conductivity $(\lambda)$. These equations correlate the measured signal $(\Delta T)$ with the conductance only when $\tau$ is greater than $4 C / k$, and for $\tau$ much greater than the system relaxation time $4 C / k, \tan h(k \tau / 2 C) \sim 1$, and so the amplitude of the signal becomes independent of $C$ leading to steady state limit.

\section{Results}

Figure 4 presents the thermal conductivity data of stainless steel 304, in the temperature range $77 \mathrm{~K}-300 \mathrm{~K}$. The experiment on stainless steel 304 was done using both steady state and pulse techniques. The values of thermal conductivity obtained by both methods agree to each other and also to those reported in the literature (Goldratt and Greenfield 1978).

The accuracy involved in the measurement of thermal conductivity using various techniques, is being estimated from further systematic measurements on NBS materials
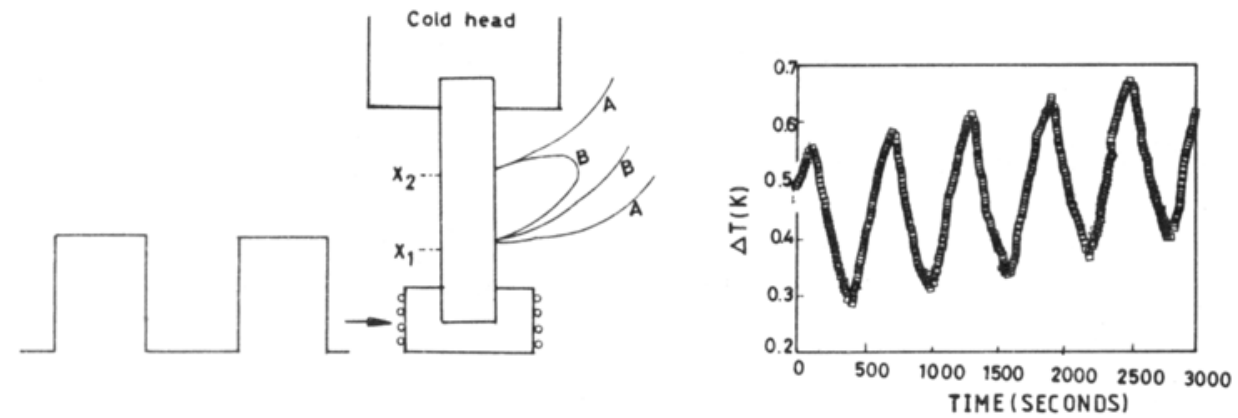

Figure 3. Schematic presentation of the pulse method. 


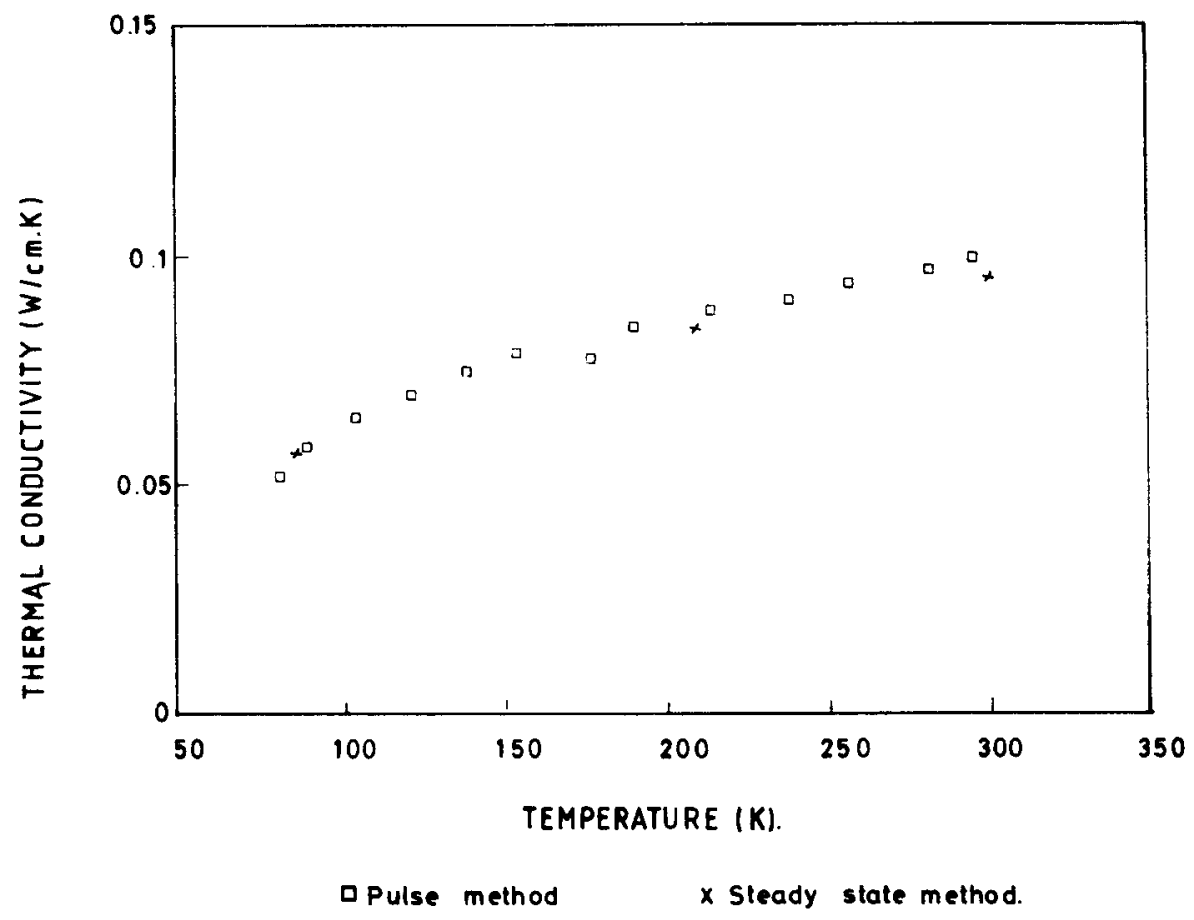

Figure 4. Thermal conductivity data on stainless steel 304 in the temperature range $77 \mathrm{~K}$ $300 \mathrm{~K}$.

(Hust and Lankford 1984): (1) Stainless steel (SRM:1461) and (2) Electrolytic Iron (RM:8420)

The experimental set up described in this paper can also be conveniently used for the determination of thermal conductivity of poor conductors such as polymers (polyethylene, polyvinyl chloride etc.) and foam insulation such as polyurethane. The results obtained on these polymeric materials will be published elsewhere.

\section{Acknowledgement}

The authors wish to thank Prof. P Radhakrishna and Ahmed Sayeed for useful discussions, the ISRO-IISc Space Technology Cell for financial support and also Dr K Ramamurti for his help.

\section{References}

Goldratt E and Greenfield A J 1978 Rev. Sci. Instrum. 491531

Hust J G and Lankford A B 1984 Nat. Bur. Stand. Special Publications pp 260-90

Maldonado O 1992 Cryogenics 32908 